\title{
Blockchain Technology as an Approach for Data Marketplaces
}

\author{
Sebastian Lawrenz \\ Institute for Software and Systems \\ Engineering, Clausthal University of \\ Technology \\ Arnold-Sommerfeld-Straße 1 \\ 38678 Clausthal-Zellerfeld, Germany \\ +495323 72-7176 \\ Sebastian.lawrenz@tu- \\ clausthal.de
}

\author{
Priyanka Sharma \\ Institute for Software and Systems \\ Engineering, Clausthal University of \\ Technology \\ Arnold-Sommerfeld-Straße 1 \\ 38678 Clausthal-Zellerfeld, Germany \\ Priyanka.sharma@tu- \\ clausthal.de
}

\author{
Andreas Rausch \\ Institute for Software and Systems \\ Engineering, Clausthal University of \\ Technology \\ Arnold-Sommerfeld-Straße 1 \\ 38678 Clausthal-Zellerfeld, Germany \\ +495323 72-8232 \\ Andreas.rausch@tu- \\ clausthal.de
}

\begin{abstract}
In the digital Economy 'Data is the new oil. In the last decade technology has disrupted every filed imaginable. One such booming technology is Blockchain. A blockchain is essentially a distributed database of records or public ledger of all transactions or digital events that have been executed and shared among participating parties. And once entered, the information is immutable. Ongoing projects and prior work in the fields of big data, data mining and data science has revealed how relevant data can be used to enhance products and services. There are uncountable applications and advantages of relevant data. The most valuable companies of today treat data as a commodity, which they trade and earn revenues.

But use of relevant data has also drawn attention by the other nonconventional organizations and domains. To facilitate such trading, data marketplaces have emerged. In this paper we present a global data marketplace for users to easily buy and share data. The main focus of this research is to have a central data sharing platform for the recycling industry. This paper is a part of the research project "Recycling 4.0" which is focusing on sustainably improving the recycling process through exchange of information. We identify providing secure platform, data integrity and data quality as some major challenges for a data marketplace. In this paper we also explore how global data marketplace could be implemented using blockchain and similar technologies.
\end{abstract}

\section{CCS Concepts}

- Information systems $\rightarrow$ World Wide Web $\rightarrow$ Web applications

$\rightarrow$ Electronic commerce $\rightarrow$ Electronic data interchange $\bullet$

Security and privacy $\rightarrow$ Systems security $\rightarrow$ Distributed systems security $\bullet$ Computer systems organization $\rightarrow$ Architectures $\rightarrow$ Distributed architectures

Permission to make digital or hard copies of all or part of this work for personal or classroom use is granted without fee provided that copies are not made or distributed for profit or commercial advantage and that copies bear this notice and the full citation on the first page. Copyrights for thirdparty components of this work must be honored. For all other uses, contact the Owner/Author.

ICBCT 2019, March 15-18, 2019, Honolulu, HI, USA

(C) 2019 Copyright is held by the owner/author(s).

ACM ISBN 978-1-4503-6268-9/19/03.

DOI: https://doi.org/10.1145/3320154.3320165

\section{Keywords}

Blockchain, Security, Data marketplaces, Data quality, Smart Contracts

\section{MOTIVATION AND INTRODUCTION}

Everyday more and more objects are getting connected to each other generating more data than ever. In particular, the Internet of Things (IoT) forms the basis for this, as sensor technology and microcontrollers are becoming cheaper and smarter [1]. Relevant data supports businesses create strong strategies and helps them making better decisions. A global data marketplace could help businesses share relevant data and help the other partners in the domain develop better-optimized products.

One major requirement for such a data marketplace is secure transactions. A user should be able to buy and sell data without worrying whether the buyer will receive legitimate data or not or whether the seller will get the payment in return. Also, a mechanism to ensure data integrity is required. Data integrity is the assurance that the data is consistent and has not been altered through the entire lifecycle. The other important requirement is that: the buyers should be able to check the quality of data before buying it. But in a data marketplace the quality has to be proven without the buyer being able to see the data. Also, the seller should not able to see the requirements of the buyer as this would reveal business strategies in some cases and the seller can also generate fake data once the patterns are known. There are many requirements and challenges for a data marketplace, but we identify the following three challenges as major challenges for a data marketplace. I.e. Secure platform, data integrity: proof that the data has not been modified at any point and ensuring data quality.

The focus of this paper is to present possible solutions for the above-mentioned challenges using blockchain and smart contracts. The rest of the paper is structured as follows. Section 2 presents a brief overview related work. In section 3 we present a scenario for a data marketplace. Section 4 presents our approach towards the major challenges identified for a data marketplace. In section 5 we show the ongoing work and next steps. And in Section 6 we summarize our conclusions and give insights for future work.

\section{STATE OF THE ART}

This section shows an overview of the state of the art at various topics discussed in this paper. 


\subsection{Data Marketplaces}

In Germany, there has already been a data marketplace for mobility data for several years, the Mobility Data Marketplace ${ }^{1}$ (MDM). In the MDM different parties could offer mobility data, such as petrol prices, or construction sites on motorways. Other parties could buy this data and use it for example for applications or as input data for navigation software. Still others only buy data to refine it and sell it again.

A similar approach was used in the European Union's Big IoT $^{2}$ research project for IoT data. This project focused on solving the interoperability of different IT systems and data structures [2].

\subsection{Blockchain Technology for Data Marketplaces}

The rise of digital technologies in the past decade has brought notable disruption in many industries. One such popularly growing technology is Blockchain. Cryptocurrencies hit the headlines after the rise of a cryptocurrency called bitcoin. As Bitcoin got more popularity the idea of decentralized currencies caught on [3]. But what makes bitcoin so special and powerful is its underlying technology- Blockchain. A blockchain is essentially a distributed database of records or public ledger of all transactions or digital events that have been executed and shared among participating parties. Each transaction in the public ledger is verified by consensus of a majority of the participants in the system. And, once entered, the information is immutable [4]. But the use case of blockchain is beyond cryptocurrencies. Many industries can be transformed and disrupted by blockchain. What makes blockchain so influential are the benefits that it inherits. The benefits of blockchain include more transparency, enhanced security, improved traceability, efficiency and reduced costs.

Another revolutionizing technology are Smart Contracts. They are self-executing scripts residing on the blockchain. When a preconfigured condition in a smart contract among participating entities is met then the parties involved in a contractual agreement can be automatically make payments as per the contract in a transparent manner [4].[5], [6].

An approach for a data marketplace based on blockchain technology was developed by the Center for Cyber-Physical Systems and Internet of Things from the University of Southern California. They implemented a prototype based on the Ethereum project3. For the problems with trust and data quality they propose a rating system for the buyers and sellers based on smart contracts. In their approach the parties rate each other after a transaction and this rating is stored in the blockchain [7].

\subsection{Data Quality}

As mentioned earlier one big challenge for a data marketplace is ensuring data quality. The buyer should be able to check the quality of data before buying it. To meet this challenge of ensuring data quality we introduce and discuss the term data quality in detail in this chapter. It is very important for to differentiate the terms information and data as they are often mixed with each other.

Data: data are just symbols, just as a list of String or integer values for example [8], [9].

\footnotetext{
${ }^{1} \mathrm{cf}$. https://www.mdm-portal.de/en/

2 cf. http://big-iot.eu/
}

Information: data that are processed to be useful, providing answers to 'who', 'what', 'where', and 'when' questions [8], [9].

Then follows that information are the interpretation of data and accordingly always partly subjective and use case dependent.

For a data trade, the quality of the data is one of the most important aspects. Therefore, this section deals with the question: What is data quality and how can we define it?

In the literature a common definition defines "data quality" as data that are fit for use by data consumers[10]. But what does "Data quality" exactly mean for the user in a data marketplace? Richard Y. Wong and his team created a catalogue based on their studies describing the criteria for data quality [10], [11], [12]. Some of these criteria are accuracy, relevancy and believability [10]. In Germany the all these criteria was clustered by the "Deutsche Gesellschaft für Informations- und Datenqualität e.V." into four categories (System supported, inherent, purpose dependent, presentation-related) using 15 criteria's [13].

The disadvantage of this clustering is that information and data quality are mixed together. This is a problem currently under research [14]. In this paper we address data quality in case of data trading so criteria's like availability are not relevant. Hence, we categorize data quality only as objective and subjective criteria's (see figure 1).



Figure 1. Categorization of data quality in objective and subjective criteria.

The objective criteria describe the content and completeness of the data set. Such objective properties can be clearly described by a suitable algorithm. But the subjective criteria, on the other hand depends on the buyer's application. Subjective properties can only be described by the buyer as it is very specific to his/her requirement. The challenge that arises here is the question of how the buyer can check these subjective criteria without revealing his business model to the seller.

In this section we presented some existing data marketplaces, and the current state of blockchain technology. We also address the already existing data marketplaces based on blockchain. While

\footnotetext{
${ }^{3} \mathrm{cf}$. https://www.ethereum.org/
} 
prior and ongoing researches have shown solutions for a implementing a data marketplace and providing a communication medium for the stakeholders, we identify ensuring data quality as one of the major challenges. In this paper we present our approach towards this problem and also present an architecture for a data marketplace.

\section{SCENARIO AND PROBLEMS}

This paper is a further development from the project "Recycling 4.0" managed by "European Regional Development Fund". The project Recycling 4.0 describes the idea of sustainably and improving the recycling process through exchange of data [15]. One way for the recycling industry to increase the recycling the recycling rate and improve efficiency is by sharing their data with each other. This creates a need for a data marketplace where the stakeholders of the domain recycling can securely sell and buy data. Thus, the research project and the paper focus on recycling 4.0 as a scenario for data marketplace.

\subsection{Recycling 4.0 as a Scenario for Data Marketplace}

One big challenge faced by the recycling industry is the loss of data during the whole product lifecycle. Degraded products, production and postconsumer waste are less well tracked, this leads to loss of valuable data, especially if products and lifetime chains are complex [15]. Therefore, a global information marketplace for recycling 4.0 can revolutionize the whole process.

There can be various challenges for implementing a data marketplace for recycling 4.0 , but we consider the already mentioned challenges as major challenges for a data marketplace in recycling 4.0 too. The challenges are:

1. Establishment of a secure platform: Providing a secure platform for transactions.

2. Establishment of data integrity: Ensuring that the source of the data is legitimate and has not been modified.

3. Ensuring data quality: The buyer should be able to check the quality of data before buying it. E.g. the buyer wants to write a program to predict the condition of electric car batteries using a data set that contains information about transaction criteria in electric cars. In particular, he/she wants to rely on data about the state of charge (frequency and type of charging station), as well as information about the driver's profile (sporty, moderate, etc.). On one hand he/she could ask the seller whether the data set contains all this information, but they would risk giving away parts of their concept to the seller. The ideal case for this trade would be that: The buyer is not able to see the data set before buying the data set but also able to check the quality of the dataset.

In the next section we present our approach towards the problem's secure platform, data integrity and data quality.

\section{SOLUTIONS AND APPROACH}

This section presents our approach towards the identifies challenges. We also present an architectural overview of how a data marketplace could work.

\subsection{Stakeholders in a Data Marketplace}

The fundamental stakeholders of a data marketplace are the sellers and the buyers. The buyers and sellers should be able to easily sell and buy data via the marketplace.

Buyer: We assume that there are a set of buyers interested in buying data from a data marketplace. E.g. the buyers can be stakeholders from the recycling domain who can use the data to enhance their products and the recycling process.

Seller: We suppose that there are sellers who are ready to sell their data in the marketplace.

\subsection{Traditional Marketplaces vs. Future Marketplaces \\ Traditional marketplaces}

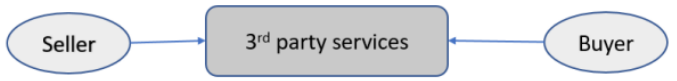

2. Buyer

Future marketplaces

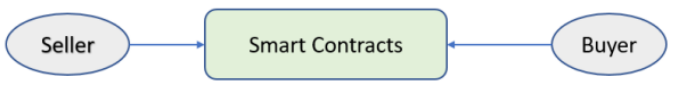

Figure 2. Traditional vs Future marketplaces.

For trading and businesses, what makes the future marketplaces different is the elimination of third parties for trading. Until now we have always required a third party that offers a platform to sell the products and the same goes for buying. In other case the seller would have his/her own platform to sell products. But in both of the above-mentioned cases, people trust the services or the people offering them as they feel they have a contract with them. In other case the seller would have his/her own platform to sell products. But in both of the above-mentioned cases, people trust the services, the people offering these services or they feel they have a contract with them. A contract is a promise or set of promises that are legally enforceable and, if violated, allow the injured party access to legal remedies [16]. In the future this trust can be build using selfexecuting, self-enforcing contracts- Smart contracts. [4]

\subsection{Approach and Possible Solutions}

Fig 3. shows our approach towards the main challenges identified I.e. providing Secure platform, ensuring data integrity and data quality. We identify that all of the required properties cannot be fulfilled by one mechanism. Thus, we divide the problems in the scope of the data marketplace in three parts.

1. Data sets- The data sets are the actual data which has to transferred to the buyer. The challenges associated with the transfer of the actual data is providing data integrity and a secure transfer. One big advantage of using blockchain is that it can be used to provide a tamper proof as any data on the blockchain is immutable. Thus, the integrity of the data can be verified if it was on blockchain. One possibility is to save the time stamps of the datasets on blockchain so that they cannot be tampered. How the data will be stored on a blockchain and how will it be transferred to the seller is a part of our ongoing project. We realize that using blockchain for our actual data transfer can be useful in many ways, the implementation possibilities will be a part of our future work.

The second problem which has been categorized is data quality.

2. Data quality- The buyer should be able to check the quality of data without seeing the actual data and the seller should not be able to see the buyer's requirement either. We identify this as one of the major challenges in the data marketplace. For checking the quality of the datasets, we propose a product quality service. This service uses a data quality check algorithm for checking the data quality. The detail explanation of how a data quality algorithm could work 
is explained in detail later in this paper. But the execution of the data quality algorithm is a part of our future work. The data quality algorithm serves as a parameter for a transaction but we identify that its scope is outside the smart contracts.

3.Transactions- We identify that the transaction i.e. approval to buy the data and receiving money are outside the scope of sending the actual data. Hence, we differentiate the actual data transfer and the transaction. The challenge associated with the transaction is that there is a requirement of a secure platform also the parties involved has to trust each other as the transaction may be tampered or denied later. For this problem we propose smart contract as a medium for the transactions. A smart Contract has self-executing scripts that reside on the blockchain. When a pre-configured condition in a smart contract among participating entities is met then the parties involved in a contractual agreement can be automatically made payments as per the contract in a transparent manner [4]. Smart contract facilitates transparency of transaction to all concerned parties as once executed its immutable.

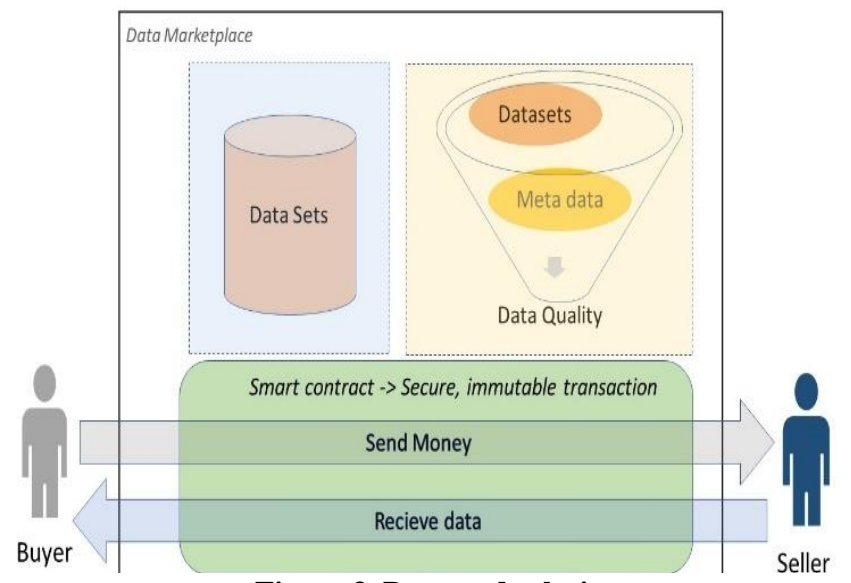

Figure 3. Proposed solution.

Hence as shown in the fig 3 . we propose different blockchain based solutions for various challenges identified for a data marketplace. Although we identify the advantages of using blockchain and similar technologies for this challenge the implementation details will be a part of our future work.

In the next section we present an architectural overview of how a data marketplace could work. We categorize the various functional requirements of the users as Services.

\subsection{Architectural Overview for Data Marketplace.}

Fig 4. shows the architectural overview of a decentralized marketplace. Various functional requirements are offered as services. The architecture shows the interaction of the fundamental stakeholders with the marketplace. The most important services for the buyers and sellers are the possibility to post, sell and buy data easily. The posting of data to be sold is done by the selling service. A seller is required to provide meta data about the data, once this information is provided, the seller can post his offer.

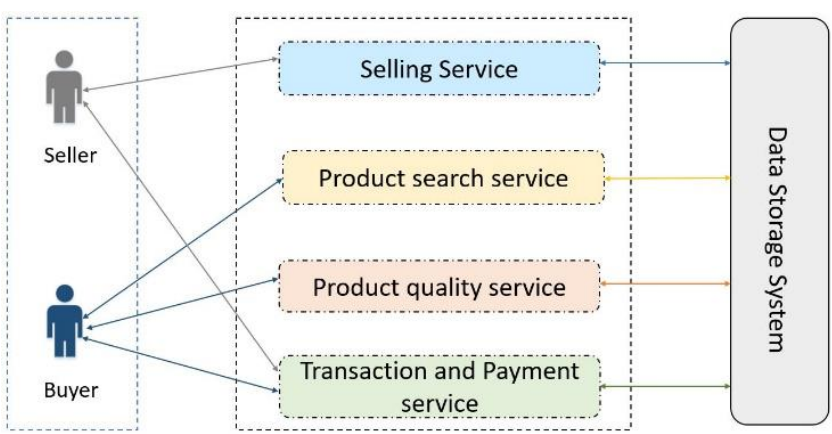

Figure 4. Architectural overview for data marketplace.

When a buyer wants to buy some data, he/she can search for it using the sematic search in the product search service, but unlike other commodities that can be bought online data cannot be returned after seeing it. For this there is a product quality service proposed. The product quality service takes the specific and subjective requirements of the buyer and checks them without the buyer being able to see the actual data. The transaction and payment service are based on a smart contract and cryptocurrency. Once the buyer and seller agree on a trading the transaction will be carried out using a smart contract. The detailed description of the functionalities of the above-mentioned services is shown in the next section.

\section{Selling Service}

The most important service for seller in the data marketplace is being able to post the data for selling easily. In order to post the data for selling the seller has to first fill up a product description template. The product description template lets the seller provide important information the about the data. In our solution we also propose a feature to generate the meta data automatically. Once the meta data is provided by the seller or the seller decides to generate is automatically using the data marketplace, the offer can be posted for selling.

\section{Product search service}

The product search service lets the buyer search for relevant data easily. In the proposed solution the buyers search is queried using "sematic search" and the most relevant posting is provided. In a small data marketplace, the buyer could browse through all the results and read the product description. But the same approach does not scale for a bigger data marketplace. There could be buyers who need more advanced search as they are looking for very specific data. For this we propose a feature "Specific Search". The specific search lets the buyer search very specific data with the help of a specification template. We will address this in more detail in future work.

\section{Product quality service}

The buyers should be able to check the quality of data before buying it. As shown in section 2.3 the subjective properties for the data quality are specific to the buyer's requirement and can vary amongst different buyers. Many users would not want to reveal their specific requirements i.e. the subjective properties of data as this might reveal their business models. We address this problem in our approach using a data quality algorithm. The data quality algorithm is used when the buyer decides to have a "specific search" which is checking the data quality for the subjective data quality. For a semantic search the product description serves as a quality assurance method. The quality search algorithm matches the specification i.e. the subjective properties provided by the buyer with the meta data and the data to provide the accuracy of the search. After the buyer requests for a specific search he/she is provided 
with more specific results. The most accurate posting can be selected. The transaction on the data marketplace is carried out in a smart contract as shown in Fig.5.

\subsubsection{Transaction and payment service}

The data marketplace uses smart contracts for the transaction and payment service. The scenario for the smart contract execution is shown in fig.5. One possibility is to build the smart contracts on the Ethereum


Figure 5. Scenario for a successful data transaction.

In this paper we outline the architectural and functional overview of a data marketplace. We will address the implementation details in future work.

\section{ON-GOING WORK AND NEXT STEPS}

Currently we are evaluating the public and private blockchains for overcoming the challenges mentioned in the above sections. Once an architecture to facilitate the data marketplace is finalized the data marketplace for the recycling domain will be implemented. The implementation details will be part of our future work, but already existing databases based on blockchain such as BigChainDB ${ }^{4}$ could be one solution. The proposed data quality check algorithm as part of product quality service is also a part of the future work.

\section{CONCLUSION}

In this work we presented the advantages of using Blockchain technology for data marketplaces. We also presented the Recycling 4.0 as the main use case for a data marketplace. The main focus of this paper is to present possible solutions for three major challenges I.e. Secure platform, data integrity and data quality. We identify that whereas blockchain and the technologies powered by blockchain can solve these challenges, there is not a common solution for these challenges. Hence, we propose smart contracts as a medium for secure transactions, and blockchain as a possibility for the actual data transfer.

\section{ACKNOWLEDGMENTS}

This paper evolved of the research project "Recycling 4.0" (digitalization as the key to the Advanced Circular Economy using the example of innovative vehicle systems) which is funded by the European Regional Development Fund (EFRE | ZW 6-85017297) and managed by the Project Management Agency NBank.

\section{REFERENCES}

[1] L. Atzori, A. Iera, and G. Morabito, "The Internet of Things: A Survey," Comput. Netw., vol. 54, no. 15, pp. 2787-2805, 2010.

[2] A. Bröring et al., "Enabling IoT Ecosystems through Platform Interoperability," IEEE Softw., vol. 34, no. 1, pp. 54-61, Jan. 2017.

[3] A. M. Antonopoulos, Mastering Bitcoin: Unlocking Digital Crypto-Currencies, 1st ed. O'Reilly Media, Inc., 2014.

[4] B. Bitcoin et al., "BlockChain Technology," 2015.

[5] G. W. Peters and E. Panayi, "Understanding modern banking ledgers through blockchain technologies: Future of transaction processing and smart contracts on the internet of money," New Econ. Wind., pp. 239-278, 2016.

[6] A. Kosba, A. Miller, E. Shi, Z. Wen, and C. Papamanthou, "Hawk: The Blockchain Model of Cryptography and PrivacyPreserving Smart Contracts," in Proceedings - 2016 IEEE Symposium on Security and Privacy, SP 2016, pp. 839-858, 2016.

[7] G. S. Ramachandran, R. Radhakrishnan, and B. Krishnamachari, "Towards a Decentralized Data Marketplace for Smart Cities," 2018.

[8] M. Chen et al., "Data, Information, and Knowledge in Visualization," IEEE Comput. Graph. Appl., vol. 29, no. 1, pp. 12-19, Jan. 2009.

[9] R. L. Ackoff and AckoffR.L., "From data to wisdom," J. Appl. Syst. Anal., vol. 16, no. 1, pp. 3-9, 1989.

[10] R. Y. Wang and D. M. Strong, "Beyond Accuracy: What Data Quality Means to Data Consumers," 1996.

[11] Y. Wand and R. Y. Wang, "Anchoring Data Quality Dimensions in Ontological Foundations," Commun. ACM, vol. 39, no. 11, pp. 86-95, Nov. 1996.

[12] D. M. Strong, Y. W. Lee, and R. Y. Wang, "Data quality in context," Commun. ACM, vol. 40, no. 5, pp. 103-110, 1997.

[13] J. P. Rohweder, G. Kasten, D. Malzahn, A. Piro, and J. Schmid, "Informationsqualität --- Definitionen, Dimensionen und Begriffe," in Daten- und Informationsqualität: Auf dem Weg zur Information Excellence, K. Hildebrand, M. Gebauer, H. Hinrichs, and M. Mielke, Eds. Wiesbaden: Vieweg+Teubner, pp. 25-45, 2008,.

[14] D. Dominique Fehrenbacher and M. Helfert, "Contextual Factors Influencing Perceived Importance and Trade-offs of Information Quality," 2012.

[15] C. Knieke, S. Lawrenz, M. Fröhling, D. Goldmann, and A. Rausch, "Predictive and flexible Circular Economy approaches for highly integrated products and their materials as given in E-Mobility and ICT," Circ. Econ. Mater. Components E-mobility - CEM ${ }^{2}, 2018$.

[16] A. Schwartz, R. E. Scott, and R. E. Scottt, "Contract Theory and the Limits of Contract Law Recommended Citation Article Contract Theory and the Limits of Contract Law," 2003

\footnotetext{
${ }^{4}$ cf. https://www.bigchaindb.com/
} 